\title{
CONTRIBUIÇÕES DA RESIDÊNCIA EM PLANEJAMENTO E GESTÃO EM SAÚDE NO ÂMBITO DISTRITAL DA VIGILÂNCIA SANITÁRIA
}

\author{
CONTRIBUTIONS OF RESIDENCY IN HEALTH \\ PLANNING AND MANAGEMENT WITHIN THE \\ DISTRICT CONTEXT OF HEALTH SURVEILLANCE
}

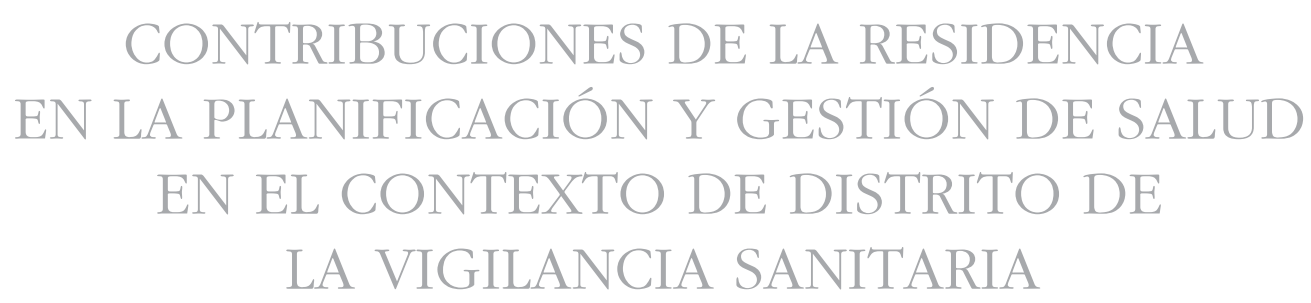

Aline Almeida Dantas ${ }^{1}$

Mariluce Karla Bomfim de Souza ${ }^{2}$

Como citar este artigo: Dantas AA, Souza MKB. Contribuições da Residência em Planejamento e Gestão em Saúde no âmbito distrital da Vigilância Sanitária. Rev baiana enferm. 2020;34:e34905.

Objetivo: discutir as contribuições da Residência Multiprofissional em Planejamento e Gestão para a formação e atuação em Vigilância Sanitária com base em um relato de experiência. Método: vivência dentro da Vigilância Sanitária em um dos 12 distritos sanitários do município de Salvador, Bahia, Brasil, nos meses de julho a setembro de 2019. Resultados: foi possível experienciar diversas atividades do setor destacando entre elas a própria rotina do setor no que se refere à emissão de alvarás sanitários, posterior aos processos de inspeção/fiscalização dos estabelecimentos/unidades de saúde e a elaboração do Relatório das Condições Sanitárias das Unidades. Conclusão: a inserção de um residente dentro da Vigilância Sanitária, além de contribuir para o serviço, destacou a potencialidade da atuação conjunta entre a Residência e a Vigilância Sanitária.

Descritores: Vigilância em Saúde. Vigilância Sanitária. Planejamento em Saúde. Formação Profissional em Saúde.

Objective: to discuss the contributions of the Planning and Management Multiprofessional Residency for the training and actions in Health Surveillance based on an experience report. Method: experience within the Health Surveillance in one of the 12 health districts of the city of Salvador, Babia, Brazil, in the months from July to September 2019. Results: various activities were experienced in the sector, with emphasis on the sector's own routine in relation to the issuance of health charters, subsequently to the processes of inspection/monitoring of health establishments/units and the elaboration of the Report on Health Conditions of the Units. Conclusion: the intern's insertion in the Health Surveillance, in addition to contributing to the service, highlighted the potential of joint action between the Residency and the Health Surveillance.

Descriptors: Public Health Surveillance. Health Surveillance. Health Planning. Health Human Resource Training.

\footnotetext{
Enfermeira. Residente do Programa de Residência Multiprofissional em Saúde Coletiva da Universidade Federal da Bahia. Salvador, Bahia, Brasil. alinedantas25@gmail.com. https://orcid.org/0000-0003-0784-280।.

2 Enfermeira. Doutora em Saúde Pública. Professora Adjunta da Universidade Federal da Bahia. Salvador, Bahia, Brasil. marilucejbv@yahoo.com.br. orcid 0000-0002$7895-4432$.
} 
Objetivo: analizar las contribuciones de la Residencia Multiprofesional en Planificación y Gestión en la formación y las acciones de Vigilancia Sanitaria con base en un relato de experiencia. Método: experiencia en la Vigilancia Sanitaria en uno de los 12 distritos sanitarios de la ciudad de Salvador, Babia, Brasil, en los meses de julio a septiembre de 2019. Resultados: se pudo disfrutar de varias actividades en el sector, destacando la propia rutina del sector en relación con la expedición de los permisos sanitarios, posterior a los procesos de inspección y supervisión de los establecimientos/unidades sanitarias y el Informe de las Condiciones Sanitarias de las Unidades. Conclusión: la inserción de un residente dentro de la Vigilancia Sanitaria, además de contribuir al servicio, destacó el potencial de acción conjunta entre la Residencia y la Vigilancia Sanitaria.

Descriptores: Vigilancia en Salud. Vigilancia Sanitaria. Planificación en Salud. Capacitación de Recursos Humanos en Salud.

\section{Introdução}

As primeiras ações sistemáticas e regulamentadas de Vigilância Sanitária no país surgiram na década de 70, em consonância com a reformulação do Ministério da Saúde, em resposta à conjuntura política, social e econômica vivida na época, destacando-se aqui a criação do Sistema Nacional de Vigilância Sanitária (SNVS). Nas décadas que se seguiram, 80 e 90, a Vigilância Sanitária, passou por processos organizacionais influenciados pelo cenário nacional que culminaram com a criação da Agência Nacional de Vigilância Sanitária (ANVISA) que perpetua até os dias atuais ${ }^{(1-2)}$.

Outro marco importante do processo de consolidação da vigilância sanitária no Brasil é a Política Nacional de Vigilância em Saúde (PNVS), instituída pela Resolução n. 588, de 2018, definida em seu artigo $3^{\circ}$, como a articulação de saberes e práticas que abrangem a Vigilância Epidemiológica, Vigilância Ambiental, Vigilância Sanitária e Saúde do Trabalhador ${ }^{(3)}$.

Tendo em vista tais saberes e práticas, estudo $^{(4)}$, pautado na definição de participação social e democracia, propõe linearidade desses dois componentes, definindo novas formas de se relacionar no ambiente do trabalho em saúde, ressaltando a importância do diálogo entre profissionais e destes com a população.

Cabe ressaltar que o conjunto das vigilâncias - epidemiológica, ambiental, sanitária e saúde do trabalhador - de acordo com a Lei Orgânica n. 8.080, de 19 de setembro de 1990, artigo 6º, constitui-se como campo de atuação do Sistema Único de Saúde (SUS) ${ }^{(5)}$.
Trazendo as particularidades da Vigilância Sanitária (VISA), o marco legal citado a refere como um "conjunto de ações capaz de eliminar, diminuir ou prevenir riscos à saúde e de intervir nos problemas sanitários decorrentes do meio ambiente, da produção e circulação de bens e da prestação de serviços de interesse da saúde abrangendo: o controle de bens de consumo e da prestação de serviços, que direta ou indiretamente, se relacionem com a saúde"(5:2).

Em outro estudo ${ }^{(6)}$, a Vigilância Sanitária é definida como um conjunto de saberes que englobam várias áreas do conhecimento além de práticas multiprofissional e institucional, que objetiva a promoção e a proteção da saúde, e busca promover qualidade e segurança, bem como eficácia e efetividade nos serviços, produtos e processos.

A VISA tem sua atuação também pautada em um conjunto de atividades, quais sejam: regulamentações concedidas por meio de licenças sanitárias para produção e comércio de bens e serviços, registro de produtos para fabricação e consumo, certificação de boas práticas de produção, monitoramento da qualidade de produtos e serviços, fiscalização do cumprimento das normas, comunicação e educação sobre riscos e vigilância de eventos adversos relacionados a esses bens ${ }^{(7)}$.

A VISA assume um caráter regulatório, mediante uma noção mais normativa e autorizativa de suas ações, tais como emissão de registros, licenciamentos e autorizações, bem como inspeções e notificações, uma vez que essas atividades 
podem gerar diversas intervenções ${ }^{(8)}$. No entanto, existem algumas contradições acerca do papel da VISA já que suas ações, pautadas na regulação, fiscalização e controle não se enquadram no conceito atual de Vigilância Sanitária como campo de atuação em saúde pública ${ }^{(9)}$.

Cabe destacar que, em outros países, no Manual de Vigilância Sanitária, da Organización Panamericana de la Salud, a VISA engloba diretamente questões relacionadas ao ambiente, abrangendo programas de saúde ambiental, abastecimento de água potável, disposição de excrementos, resíduos sólidos, assentamentos, saneamento de estabelecimentos de saúde e outras instituições e saneamento em desastres ${ }^{(10)}$.

Portanto, diante do amplo escopo de atuação da Vigilância Sanitária no Brasil, a Resolução da Diretoria Colegiada (RDC) n. 50/2002 reafirma que a VISA também atua no planejamento, programação, elaboração e avaliação de projetos físicos, inclusive de estabelecimentos assistenciais de saúde, bem como na sua fiscalização ${ }^{(11)}$.

Nesse sentido, o Programa de Residência Multiprofissional em Saúde Coletiva com Área de Concentração em Planejamento e Gestão em Saúde, do Instituto de Saúde Coletiva da Universidade Federal da Bahia (ISC/UFBA), iniciado em 2016, que tem como objetivo a especialização por meio da educação em serviço, e tomando-se como referência o exposto em estudo ${ }^{(6)}$ a respeito do entendimento da VISA, reconhece a importância desse serviço no processo de formação profissional que permite ao residente a vivência em diferentes espaços.

Cabe ressaltar que o referido programa vem, ao longo desses anos, desenvolvendo suas atividades em setores da Secretaria Municipal de Saúde de Salvador (SMS/SSA), na Secretaria Estadual de Saúde da Bahia (Sesab) e em nove distritos sanitários, dentre os doze que compõem o território em saúde da capital baiana, Salvador. Tendo em vista que o curso tem duração de dois anos, no primeiro ano, os residentes são inseridos nas sedes dos distritos sanitários, e no segundo ano, eles são distribuídos em diversos setores da SMS e Sesab.

Contudo, essa vivência dentro da VISA serviu de base para a elaboração desse Relato de
Experiência partindo da riqueza e do aprendizado adquirido durante os dois meses dentro do setor.

Este artigo tem por objetivo discutir as contribuições da Residência Multiprofissional em Planejamento e Gestão para a formação e atuação em Vigilância Sanitária com base em um relato de experiência.

\section{Método}

Tomando-se como ponto de partida o Programa de Residência Multiprofissional em Saúde Coletiva com Área de Concentração em Planejamento e Gestão em Saúde, do Instituto de Saúde Coletiva da Universidade Federal da Bahia (ISC/ UFBA), experiências têm sido vivenciadas por estudantes residentes sob a supervisão de docente e acompanhamento de preceptoria, em particular no ano de 2019, recorte a que se refere este relato.

Em março de 2019, teve início a quarta turma de residentes do programa, composta por 18 profissionais com formação nas áreas de enfermagem, fonoaudiologia, fisioterapia, saúde coletiva, psicologia, nutrição e odontologia, os quais foram divididos em trios e alocados para as atividades em serviço dentro dos distritos sanitários, dentre eles o distrito sanitário de Brotas, local onde ocorreu a vivência que originou este relato. O distrito possui uma extensão territorial de $11,25 \mathrm{~km} 2$, população de 217.100 hab. (2018) e densidade demográfica de 19.297,7 hab/km², sendo, portanto, o terceiro distrito mais povoado do município de Salvador ${ }^{(12-13)}$.

Após a entrada no distrito, com acolhimento do preceptor e acompanhamento sistemático da supervisora docente, decidiu-se pelo desenvolvimento de ações de planejamento, programação e acompanhamento do cotidiano do processo de trabalho nos setores que compõem a estrutura física nas sedes distritais, a saber: ações; informação; vigilância epidemiológica, vigilância sanitária. Com referência a este último, e tendo em vista a pouca aproximação em vivências formativas anteriores, inclusive na graduação, viu-se a necessidade de conhecer as suas demandas e especificidades da atuação. 
Cabe ressaltar que concomitantemente à inserção dos residentes nos setores, dentre eles a VISA, houve a elaboração de um plano de trabalho de forma conjunta entre o residente, o supervisor, o preceptor e a referência técnica do respectivo setor que contemplava: início e término do estágio, ação/atividade, objetivo e meta/produto. O plano foi desenvolvido com base nas demandas do setor naquele momento, dialogando com as necessidades de aprendizagem do residente no que se refere à formação em serviço e para o serviço.

Assim, a vivência dentro do setor da VISA, no âmbito distrital, foi no período de 1 de julho a 30 de setembro de 2019, 3 dias por semana, totalizando uma carga horária de 288 horas no serviço. Além da imersão no processo de trabalho proposto, foram sugeridas ações de planejamento. No conjunto de atividades desenvolvidas, enfatiza-se o processo de elaboração de um "Relatório sobre as Condições Sanitárias das Unidades de Saúde" sob o controle da VISA distrital.

Para a realização das atividades no setor foram acessados documentos diversos, dentre diretrizes, manuais, notas técnicas e resoluções das diretorias colegiadas (RDC). Especificamente para a produção do relatório, foram consultadas (os): Diretrizes para estruturação de Farmácias no âmbito do Sistema Único de Saúde, 2009; Manual da Estrutura Física das Unidades Básica de Saúde-Saúde da família, Ministério da Saúde, 2a ed., 2008; Manual de Normas e Procedimentos para Vacinação, 2014; Manual de Normas Técnicas e Rotinas Operacionais do Programa de Triagem Neonatal, 2002; Nota Técnica n. 217/2011-D-DST-AIDS-HV/SVS/MS, que trata de informações sobre a temperatura de armazenamento e transporte dos kits de testes rápidos para HIV e sífilis; Portaria 453, de 1 de junho de 1998, que aprova o Regulamento Técnico que estabelece as diretrizes básicas de proteção radiológica em radiodiagnóstico médico e odontológico, dispõe sobre o uso dos Raios X e diagnósticos em todo o território nacional e dá outras providências; RDC 50/2002, que dispõe sobre o regulamento técnico para planejamento, programação, elaboração e avaliação de projetos físicos de estabelecimentos assistenciais de saúde; RDC 63/2011, que dispõe sobre os requisitos de boas práticas de funcionamento para os serviços de saúde; RDC 15/2012, que dispõe sobre os requisitos de boas práticas para o funcionamento de produtos para saúde e dá outras providências; RDC 306/2004, que dispõe sobre o regulamento técnico para o gerenciamento de resíduos no serviço de saúde.

\section{Resultados}

No processo de trabalho vivenciado nos três meses, observou-se que fazem parte da rotina da equipe, atividades de inspeção, fiscalização e atividades orientativas/educativas aos proprietários que buscam o serviço, a fim de ter orientações sobre procedimentos de abertura, autorização e funcionamento do seu estabelecimento. No entanto, enquanto residente durante os três meses, não foi possível acompanhar a inspeção em tais serviços ou estabelecimentos, justificado por questões de sigilo profissional, segundo informações da coordenação do setor.

Entretanto, internamente, foi possível vivenciar o cotidiano, com acompanhamento de abertura de processo para obtenção de Alvará Sanitário, inclusive aquelas motivadas por solicitações de órgãos parceiros e denúncias diversas, bem como a emissão de diagnóstico situacional, e posterior adoção de medidas para correção/adequação às normas sanitárias vigentes com emissão de termos próprios, tais como notificações.

Embora os procedimentos para emissão de diagnóstico situacional e adoção de medidas para adequação às normas seja parte do cotidiano, o processo de monitoramento e avaliação dessa atividade e de outras que compõem o escopo da VISA parece requerer maior investimento, por exemplo, faz-se necessário (re)avaliar periodicamente as condições higiênico-sanitárias em que se encontram esses estabelecimentos de saúde acima relacionados.

Portanto, em conjunto com os profissionais da equipe de VISA, acordou-se como uma contribuição/produto da Residência a elaboração do 
Relatório das Condições Sanitárias das Unidades, devido a participação no acompanhamento do processo de emissão de diagnóstico e orientação e medidas. Para essa atividade, inicialmente, foi feito um levantamento dos relatórios técnicos/pareceres e notificações que compõem o arquivo do setor, sendo selecionados aqueles emitidos entre os anos de 2015 e 2019. Cabe ressaltar que a definição do recorte temporal deu-se em decorrência da disponibilidade de documentos que abrangem esse período, enquanto nos anos anteriores alguns estabelecimentos analisados não possuíam documento disponível no momento.

Após a leitura do aparato legal e normativo e do resgate de aporte teórico selecionado, tomando por base os registros disponíveis sobre os estabelecimentos, procedeu-se a descrição das condições sanitárias, higiênicas e estruturais das unidades de saúde distritais, retratando e relacionando as não conformidades encontradas, o padrão sanitário recomendado e os possíveis responsáveis para as inconsistências encontradas tanto por setor quanto num contexto mais geral. A fim de registrar e ter um panorama geral sobre as condições sanitárias das unidades de saúde elaborou-se um quadro síntese em que constava: área/setor; situação atual; situação recomendada; responsabilidade.

Dessa forma, fez-se também o levantamento da situação recomendada para cada não conformidade encontrada, tendo como base os documentos legais que dão sustentação à prática da VISA, e buscou-se relacionar também os responsáveis para correção de cada inconsistência encontrada.

Assim, observou-se que manter as boas condições higiênico-sanitárias das unidades é responsabilidade da gestão a nível municipal e distrital, bem como das equipes. Salienta-se que existem questões mais voltadas para a gestão e outras que são inerentes às equipes, visto que envolvem diretamente o processo de trabalho.

Partindo dessa visão diversificada da atuação da VISA foi possível ampliar o olhar para questões que muitas vezes passam despercebidas no âmbito da atenção básica, tais como os aspectos gerenciais das condições higiênico-sanitárias das unidades de saúde, o que foi possível com a elaboração do relatório.

Com o levantamento principalmente das não conformidades dos pareceres técnicos, pressupõe-se que muitas questões são negligenciadas no cotidiano das unidades e das equipes, questões estas que podem inferir direta e indiretamente na saúde dos usuários e no próprio processo de trabalhos das equipes. Com isso, surge a necessidade de formulação de estratégias articuladas entre a VISA e os demais setores distritais que lidam direta e indiretamente com as unidades de saúde.

\section{Discussão}

Durante o período de vivência dentro da VISA distrital foi possível experienciar diversas atividades do setor, destacando entre elas a própria rotina do setor no que se refere à emissão de alvarás sanitários, posterior aos processos de inspeção/fiscalização dos estabelecimentos/unidades de saúde e a elaboração do Relatório das Condições Sanitárias das Unidades.

Diante disso, pensar no serviço enquanto espaço de formação é essencial para a qualificação profissional de trabalhadores do SUS. Tendo em vista a sua formação, seja na gestão ou nas práticas do campo, sabe-se que o serviço tende a proporcionar inúmeras possibilidades de aprendizado $^{(14)}$. Pensando nessa diversidade de aprendizado, a VISA, enquanto setor alocado na sede do distrito, foi inserida como campo de atuação da Residência em Planejamento e Gestão.

É importante destacar que a VISA que compõe o distrito sanitário tem em seu espaço físico uma sala reservada à equipe da VISA distrital. Atualmente 17 profissionais compõem a equipe, de diversas categorias, conforme demanda este tipo de serviço e o seu objeto diverso, por considerar os bens de consumo e os serviços específicos que estão sob seu controle e fiscalização.

Atualmente, sob a supervisão da VISA distrital, além de toda demanda dos estabelecimentos existentes no território, estão sob o controle sanitário, nove unidades de saúde, das quais um 
Centro de Atenção Psicossocial, três Unidades de Saúde da Família (USF), três Unidades Básicas de Saúde (UBS) sem equipes de saúde da família, uma Unidade de Atendimento Odontológico (UAO) e uma Unidade de Pronto Atendimento 24h (UPA).

As ações da equipe da vigilância nas unidades sob sua responsabilidade sanitária objetivam mitigar riscos e agravos à saúde da população por meio do incentivo às boas práticas nos serviços de saúde. No âmbito de atuação da VISA, tais atividades se desenvolvem por meio de visitas técnicas que objetivam avaliar as condições higiênico-sanitárias, estruturais e operacionais dos estabelecimentos de interesse da saúde, bem como emissão de pareceres e/ou notificações, quando for o caso.

Nesse distrito, foi a primeira vez em que, de forma sistemática e programada, houve a imersão de residentes no setor da VISA por período determinado e acordado com a equipe. Tal fato pode explicar o certo estranhamento dos profissionais nos primeiros dias com a presença do residente.

No decorrer dos dias, foi possível perceber mais conforto da equipe com a presença constante de um residente fazendo parte de suas rotinas, principalmente à medida que foi sendo definido um produto que contribuiria tanto para o setor quanto para o processo de formação do residente, e sua construção era compartilhada com todos.

No entanto, a questão desafiadora durante esse processo deu-se, predominantemente, em decorrência do espaço físico, que não comporta adequadamente a equipe, principalmente nos momentos de reuniões e/ou planejamento, somando-se a isso, o fato de que a VISA ainda presta atendimento ao público (proprietários ou gerentes dos estabelecimentos) dentro desse espaço, fato que, por vezes, dificultou a permanência do residente dentro do setor.

Diante disso, faz-se necessário pensar na estruturação das diversas VISA locais, levando em consideração as suas particularidades e o seu processo de trabalho, o que requer ampla capacidade de articulação em rede proporcionando a formação de vínculos entre a VISA e as instituições com as quais se relaciona, bem como, da VISA com consumidores ${ }^{(15)}$.

Embora existam dificuldades, sabe-se que a relação residência-serviço é extremamente potente, pois, ao mesmo tempo em que contempla a formação em serviço do residente, este contribui para o andamento e/ou melhoria dos processos de trabalho das equipes nas quais se insere. Como exemplo disso cita-se a construção do Relatório das Condições Sanitárias das Unidades que, além de sistematizar, oferece ao serviço uma visão ampla sobre a situação sanitária das unidades de saúde, e proporciona ao residente o conhecimento sobre a dinâmica do serviço aproximando-o do conhecimento técnico, haja vista que a elaboração de tal documento requer a leitura de bases legais que regem o serviço da VISA.

Como visto, dificuldades existiram e existem, já que se está imerso num cenário real e não ideal. Entretanto, quando se pensa na formação em serviço, a VISA constitui-se uma área extremamente rica, uma vez que sua diversidade de ações a torna dinâmica e, ao mesmo tempo, complexa.

Dessa forma, este relato torna-se relevante na medida em que destaca a experiência vivida dentro da VISA e sua potencialidade enquanto campo de formação, e para que possa estimular cada vez mais a inserção desse serviço nos processos de formação profissional, haja vista sua complexidade e riqueza de ações, pautadas na multiprofissionalidade em que se articulam e se completam.

O presente relato mostra como a VISA, dentro das suas particularidades, contribui para o processo de formação do residente, ampliando o olhar deste para o setor e favorecendo um conhecimento amplificado das ações e competências de cada serviço que compõe a rede.

\section{Conclusão}

Ao vivenciar a Vigilância Sanitária dentro da Residência de Planejamento e Gestão, foi possível perceber o quanto esta passa despercebida durante os processos de formação da maioria dos cursos voltados à saúde. A inserção de um 
residente no setor não só contribuiu com o processo de trabalho da equipe, como também favoreceu a abertura e inclusão da VISA enquanto espaço de formação, destacando a potencialidade da atuação conjunta entre a Residência e a Vigilância Sanitária.

Embora o setor apresente algumas limitações, como espaço físico limitado, que dificulta as reuniões de equipe, a inclusão da VISA pela Residência, como espaço de formação, ainda assim, proporciona ao residente a ampliação do escopo de vivências durante o processo, fazendo com que este compreenda na sua totalidade como se dá o andamento dos serviços e como estes correlacionam suas ações de prevenção, promoção e proteção da saúde.

Assim, torna-se relevante a construção deste relato, pois além de destacar as limitações e potencialidades do serviço que, assim como os demais, necessita de um olhar voltado à melhoria de suas condições físicas e estruturais para melhor desempenhar suas funções, também se trata de um espaço potencialmente rico que muito tem a contribuir no processo de formação profissional, visto que suas ações são de natureza multiprofissional e articula-se com outros pontos da rede.

\section{Colaborações}

1 - concepção, projeto, análise e interpretação dos dados: Aline Almeida Dantas e Mariluce Karla Bomfim de Souza;

2 - redação do artigo e revisão crítica relevante do conteúdo intelectual: Aline Almeida Dantas e Mariluce Karla Bomfim de Souza;

3 - aprovação final da versão a ser publicada: Aline Almeida Dantas e Mariluce Karla Bomfim de Souza.

\section{Referências}

1. Luchesse G. Organização e regulamentação sanitária: os rumos da vigilância sanitária no Brasil [tese]. Rio de Janeiro (RJ): Escola Nacional de Saúde Pública Sérgio Arouca/Fiocruz; 2001 [cited 2020 Mar 7]. Available from: https://www. arca.fiocruz.br/bitstream/icict/4551/2/90.pdf
2. Seta MH. Construção do Sistema Nacional de Vigilância Sanitária: Uma Análise das Relações Intergovernamentais na Perspectiva do Federalismo [tese]. Rio de Janeiro (RJ): Instituto de Medicina Social, Universidade do Estado do Rio de Janeiro; 2007 [cited 2020 Mar 7]. Available from: https://www.abrasco.org.br/UserFiles/File/GT/ VISA/Teses/TeseMarisdefinitivacomprotecao.pdf.

3. Conselho Nacional de Saúde. Resolução n. 588, de 12 de Julho de 2018. Institui a Política Nacional de Vigilância em Saúde (PNVS) [Internet]. Brasília (DF); 2018 [cited 2019 Dez 16]. Available from: http://conselho.saude.gov.br/resolucoes/2018/ Reso588.pdf

4. Fernandes VR, Luz ZP, Amorim AC, Sérgio JV, Castro MC, Monken M, et al. O lugar da vigilância no SUS - entre os saberes e as práticas de mobilização social. Ciênc saúde coletiva. 2017 out;22(10): 317381. DOI: $10.1590 / 1413-812320172210.1772017$

5. Brasil. Lei 8.080, de 19 de setembro de 1990. Dispõe sobre as condições para a promoção, proteção e recuperação da saúde, a organização e o funcionamento dos serviços correspondentes e dá outras providências [Internet]. Brasília (DF); 1990 [cited 2019 Oct 14]. Available from: http:// www.planalto.gov.br/ccivil_03/leis/18080.htm

6. Costa EA. Fundamentos da vigilância sanitária. In: Costa EA, organizadora. Vigilância Sanitária: Temas Para Debate. Salvador: EDUFBA; 2009. p. 11-36.

7. Silva JAA, Costa EA, Lucchese G. SUS 30 Anos: Vigilância Sanitária. Ciênc saúde coletiva. 2018;23(6):1953-62. DOI: 10.1590/141381232018236.04972018

8. Seta MH, Oliveira CVS, Pepe VLE. Proteção à Saúde no Brasil: o Sistema Nacional de Vigilância Sanitária. Ciênc saúde coletiva. 2017;22(10):322534. DOI: 10.1590/1413-812320172210.16672017

9. Oliveira CM, Cruz MM. Sistema de Vigilância em Saúde no Brasil: avanços e desafios. Saúde debate. 2015;39(104):255-67. DOI: 10.1590/0103-110420151040385

10. Herderra R, Cúneo C, Zapeda F, Sáenz R. Manual de Vigilancia Sanitaria. In: Organización Panamericana de la Salud. Fundación W. K. Kellogg. Manuales Operativos PALTEX [Internet]. Washington (DC); 1996. p. 91-240 [cited 2019 Dec 11]. Available from: https://iris.paho.org/ handle/10665.2/3156

11. Agência Nacional de Vigilância Sanitária. Resolução-RDC nำ 50, de 21 de Fevereiro de 
2002. Dispõe sobre o Regulamento Técnico para planejamento, programação, elaboração e avaliação de projetos físicos de estabelecimentos assistenciais de saúde [Internet]. Brasília (DF); 2002 [cited 2019 Oct 14]. Available from: https:// bvsms.saude.gov.br/bvs/saudelegis/anvisa/2002/ rdc0050_21_02_2002.html

12. Salvador. Plano Municipal de Saúde 2018-2021. Volume II - Distritos Sanitários [Internet]. Salvador (BA); 2018 [cited 2020 Mar 8]. Available from: http://www.saude.salvador.ba.gov.br/ secretaria/wp-content/uploads/sites/2/2018/12/ Plano-Municipal-de-Sa\%C3\%BAde-2018-2021VOLUME-II_aprovado-pelo-CMS-21.11.18.pdf

13. Salvador. Secretaria Municipal da Saúde. Datasus. Tabnet - Salvador. Informações de Saúde. Estatísticas Vitais [Internet]. Salvador (BA); 2019 [cited 2020 Mar 8]. Available from: http:// www.tabnet.saude.salvador.ba.gov.br/tabcgi. exe?tabpop/populacao.def

14. Rocha AF, Breier A, Souza B, Almeida CN, Santos CM, Rohloff CC, et al. Sobre a Residência Integrada em Saúde com ênfase em Vigilância em Saúde. Ciênc saúde coletiva. 2017; 22(10):3467-72. DOI: https://doi.org/10.1590/ 1413-812320172210.18102017

15. Leal COBS, Teixeira CFS. Solidariedade: uma perspectiva inovadora na gestão e organização das ações de Vigilância Sanitária. Ciênc saúde coletiva. 2017;22(10):3161-72. DOI: 10.1590/1413-812320172210.18022017

Recebido: 18 de dezembro de 2019

Aprovado: 11 de março de 2020

Publicado: 9 de abril de 2020

A Revista Baiana de Enfermagem utiliza a Licença Creative Commons - Atribuição-NãoComercial 4.0 Internacional.

https://creativecommons.org/licenses/by-nc/4.0/

Este artigo é de acesso aberto distribuído sob os termos da Licença Creative Commons (CC BY-NC).

Esta licença permite que outros remixem, adaptem e criem a partir do seu trabalho para fins não comerciais. Embora os novos trabalhos tenham de lhe atribuir o devido crédito e não possam ser usados para fins comerciais, os usuários não têm de licenciar esses trabalhos derivados sob os mesmos termos. 